\title{
Spermine-modified Antheraea pernyi silk fibroin as a gene delivery carrier
}

\author{
This article was published in the following Dove Press journal: \\ International Journal of Nanomedicine \\ 14 March 2016 \\ Number of times this article has been viewed
}

Yanni Yu'
Yongpei Hu'
Xiufang Li'
Yu Liu'
Mingzhong Li'
Jicheng Yang'
Weihua Sheng'
'National Engineering Laboratory
for Modern Silk, College of Textile
and Clothing Engineering, Soochow
University, ${ }^{2}$ Cell and Molecular
Biology Institute, College of Medicine,
Soochow University, Suzhou,
People's Republic of China

Yanni Yu'

Yongpei $\mathrm{Hu}^{\prime}$

Xiufang $\mathrm{Li}^{1}$

Yu Liu'

Mingzhong $\mathrm{Li}^{\prime}$

Jicheng Yang ${ }^{2}$

Weihua Sheng

\begin{abstract}
for Modern Silk, College of Textile and Clothing Engineering, Soochow Biology Institute, College of Medicine, People's Republic of China
\end{abstract} National Engineering Laboratory for Modern Silk, College of Textile and Clothing Engineering, Soochow University, No 199 Ren'ai Road, Industrial Park, Suzhou 215I23, People's Republic of China Tel +86 5 I 26706 I I 50 Fax +86 5I26724 6786 Email mzli@suda.edu.cn
Correspondence: Mingzhong Li

\begin{abstract}
The development of a novel cationized polymer used as a gene delivery carrier that can conveniently and effectively transfect cells resulting in a stably expressed target gene remains a challenge. Antheraea pernyi silk fibroin (ASF) is a cytocompatible and biodegradable natural polymer, and it possesses Arg-Gly-Asp sequences but a negative charge. In order to render ASF amenable to packaging plasmid DNA (pDNA), spermine was used to modify ASF to synthesize cationized ASF (CASF), which was used as a gene delivery carrier. CASF was characterized using trinitrobenzene sulfonic acid assay, the zeta potential determination, and a Fourier transform infrared analysis, and the results of these characterizations indicated that the $-\mathrm{NH}_{2}$ in spermine effectively reacts with the $-\mathrm{COOH}$ in the side chains of ASF. Spermine grafted to the side chains of ASF resulted in the conversion of the negative charge of ASF to a positive charge. CASF packaged pDNA and formed CASF/pDNA complexes, which exhibited spherical morphology with average particle sizes of $215-281 \mathrm{~nm}$ and zeta potential of approximately $+3.0 \mathrm{mV}$ to $+3.2 \mathrm{mV}$. The results of the MTT assay, confocal laser scanning microscopy, and flow cytometry analysis in a human endothelial cell line revealed that CASF/pDNA complexes exhibited lower cytotoxicity and higher transfection efficiency compared to the pDNA complexes of polyethyleneimine. These results indicate that our synthesized CASF, a cationized polymer, is a potential gene delivery carrier with the advantages of biodegradability and low cytotoxicity.
\end{abstract}

Keywords: silk fibroin, spermine, cationized polymer, gene delivery carrier

\section{Introduction}

Gene therapy is an important approach to treat a variety of diseases. ${ }^{1}$ The principal step in gene therapy is the delivery of nucleic acids into cells. ${ }^{2}$ Gene delivery systems include both viral and nonviral delivery systems. Viral delivery systems have high gene delivery efficiency, ${ }^{3}$ but they also have disadvantages, such as immunogenicity, the possibility of permanent insertion into the host genome, and difficulties in handling. ${ }^{4}$ In contrast, nonviral delivery systems have received substantial attention because they can be structurally varied, are relatively safe, and can carry large and diverse amounts of genetic materials into cells. ${ }^{5}$ The effectiveness and biocompatibility of nonviral plasmid DNA (pDNA) delivery systems based on cationized liposomes or chemically synthesized polymers have developed over the past decade. ${ }^{6}$ Several polymeric gene delivery systems have been investigated, such as polyethyleneimine (PEI), ${ }^{7}$ poly-(L-lysine) ${ }^{8}$ chitosan, ${ }^{9}$ and dendrimers. ${ }^{10}$ Among these polymers, PEI is a typical nonviral gene delivery carrier and is often considered the gold standard of gene transfection. ${ }^{11}$ Branched PEI $(25 \mathrm{kDa})$ has produced good results in gene therapy experiments. ${ }^{12,13}$ It has a relatively high transfection efficiency based on the proton sponge effect, which can protect DNA from endosomal or lysosomal degradation. ${ }^{14,15}$ Nevertheless, PEI with a high molecular weight is cytotoxic and is not easily 
biodegradable by cells. ${ }^{16-18}$ Therefore, a key challenge for nonviral pDNA delivery systems is the development of a new cationized polymer that not only effectively transfers therapeutic DNA into the targeted cells but also exhibits biodegradability and noncytotoxicity.

Silk fibroin produced by the silkworm is a natural protein that has been widely used in tissue engineering scaffolds and as a drug delivery carrier due to its biocompatibility, bioactivity, and biodegradability. ${ }^{19,20}$ Bombyx mori silk fibroin (BSF) and Antheraea pernyi silk fibroin (ASF) are the two most extensively used silk fibroin species. BSF has positive effects on cell adhesion, viability, growth, and differentiation ${ }^{21-23}$ and has been widely used in reconstruction of skin, vascular tissue, and cartilage tissue. ${ }^{24-26}$ Compared to BSF, the amino acid composition of ASF contains abundant tripeptide sequences of Arg-Gly-Asp (RGD), which are favorable for cell adherence. ${ }^{27,28}$ In vitro investigations have shown that the use of ASF matrices results in greater cell attachment and growth than BSF matrices. ${ }^{29}$ In our previous studies, we used ASF to coat PEI/pDNA complexes and transfected ASF/ PEI/pDNA complexes into L929, HEK293, and HCT 116 cells. ${ }^{30,31}$ The results revealed that ASF reduces the cytotoxicity of PEI/pDNA complexes by shielding the excess positive charge of PEI and improves transfection efficiency. However, pDNA must be condensed by the positively charged PEI, as negatively charged ASF did not package and condense pDNA in previous studies. ${ }^{30,31}$ If cationized ASF (CASF) is synthesized by grafting compounds with low molecular weights and high densities of positive charge onto the side chains of ASF, then, in the presence of a suitable positive charge density in CASF, exogenous DNA can be packaged and transfected into cells through endocytosis. Such a gene delivery carrier would be completely biodegradable and exhibit low cytotoxicity.

Spermine is a biogenic tetraamine with two primary and two secondary amino groups. Spermine is present in all eukaryotic cells and is involved in cellular metabolism. ${ }^{32}$ It reversibly condenses DNA and is routinely used in DNA isolation. ${ }^{33} \mathrm{We}$ hypothesized that some of the negative charges on ASF can be shielded and changed to positive charges after spermine is grafted onto the side chains of $\mathrm{ASF}$, and that this process can be used to synthesize CASF. CASF can package exogenous DNA directly and exhibits advantages such as biodegradability and low cytotoxicity; therefore, CASF can be used as a gene delivery carrier. We present a novel biodegradable and bioactive CASF for use as a gene delivery vehicle through the reaction of the $-\mathrm{NH}_{2}$ in spermine with the $-\mathrm{COOH}$ in ASF side chains in the presence of 1-ethyl-(3-dimenthylaminopropyl)-3-carbodiimide hydrochloride (EDC). CASF was used to package pDNA to form $\mathrm{CASF} / \mathrm{pDNA}$ complexes, which were used to transfect a human endothelial cell line (EA.hy926) in vitro. Cytotoxicity assays, flow cytometry analysis, and confocal microscopy observation revealed that the CASF/pDNA complexes exhibited lower toxicity and higher transfection efficiency than PEI/pDNA.

\section{Materials and methods Materials}

A. pernyi silk fibers were purchased from Liaoning Province (People's Republic of China). PEI (branched; molecular weight: $25 \mathrm{kDa})$, EDC, 3-(4, 5-dimethyl-2-thiazolyl)-2, 5-diphenyl-2-H-tetrazolium bromide (MTT), ethylenediamine, and trinitrobenzene sulfonic acid (TNBS) were purchased from Sigma-Aldrich (St Louis, MO, USA). Plasmid DNA encoding enhanced green fluorescent protein (EGFP; 4,733 bp; Genbank accession number: U55762) was purchased from Clontech (Mountain View, CA, USA), amplified in the DH5 $\alpha$ Escherichia coli strain, and purified using a Qiagen Plasmid Mega Kit (Qiagen, Chatsworth, CA, USA). The concentration of plasmid DNA was determined by measuring the ultraviolet (UV) absorbance at $260 \mathrm{~nm}$. The human endothelial cell line EA.hy926 (CRL-2922 ${ }^{\text {TM}}$ ) was purchased from the American Type Culture Collection (ATCC, Manassas, VA, USA). The experiments were formally reviewed and approved by the ethics committee of Soochow University. Reviewed by the ethics committee, the experimental design and implementation have fully considered the principle of security; the experimental content did not exist potential damage and risk, and followed the principles outlined in the Declaration of Helsinki.

\section{Preparation of ASF solution}

ASF solution was prepared following the procedure described previously. ${ }^{34}$ Briefly, the $A$. pernyi silk fibers were degummed by three treatments with $2.5 \mathrm{~g} / \mathrm{L} \mathrm{Na}_{2} \mathrm{CO}_{3}$ aqueous solution at $98^{\circ} \mathrm{C}-100^{\circ} \mathrm{C}$ for $30-45$ minutes. After being rinsed and airdried at $60^{\circ} \mathrm{C}, 10 \mathrm{~g}$ of the $A$. pernyi silk fibers were dissolved in $100 \mathrm{~g}$ of melted calcium nitrate tetrahydrate at $100^{\circ} \mathrm{C}$ for 5 hours. The cooled solution was dialyzed (molecular weight cutoff: 9-12 kDa) against deionized water for 3 days to obtain the regenerated ASF solution with a concentration of $\sim 2.2 \mathrm{wt} \%$. The concentration of ASF solution was adjusted to $2.0 \mathrm{wt} \%$ with deionized water.

\section{Preparation of CASF}

First, $50 \mathrm{~mL}$ of ASF solution (2.0 wt $\%$ ) was stirred in an ice bath. Spermine was then added to the solution at $0.5 \mathrm{wt} \%$, 
$1.0 \mathrm{wt} \%, 1.5 \mathrm{wt} \%, 2.0 \mathrm{wt} \%, 4.0 \mathrm{wt} \%, 6.0 \mathrm{wt} \%, 8.0 \mathrm{wt} \%$, and $10.0 \mathrm{wt} \%$ of the ASF weight in solution. The $\mathrm{pH}$ value of the mixture was adjusted to 7.0 by the addition of hydrochloric acid solution. EDC was added to the mixture to account for $20 \mathrm{wt} \%$ of the weight of ASF in solution. The mixture was then stirred for 10-12 hours in an ice bath. Finally, the CASF solution was obtained by dialysis against deionized water at $4^{\circ} \mathrm{C}$ for 72 hours to remove excess reagents.

\section{Determination of free amino groups}

Free amino group content was determined using the TNBS assay. ${ }^{35}$ Ethylenediamine was used as a standard. Standard solutions $(0.5-7.5 \mu \mathrm{mol}, 1 \mathrm{mg} / \mathrm{mL})$ and test samples (10 mg/mL, $5 \mathrm{~mL}$ ) were prepared. To each solution, $200 \mu \mathrm{L}$ of $15 \mathrm{mg} / \mathrm{mL}$ TNBS was added and diluted to $3 \mathrm{~mL}$ with double-distilled water; then, $1 \mathrm{~mL}$ of $0.8 \mathrm{M} \mathrm{NaHCO}_{3}$ was added to the mixture. The reaction continued for 2 hours at $37^{\circ} \mathrm{C}$, followed by the addition of $3 \mathrm{~mL}$ of $1 \mathrm{M} \mathrm{HCl}$. UV absorbance was measured at $360 \mathrm{~nm}$ using a spectrophotometer (UV-3600; Shimadzu, Tokyo, Japan). The sample absorbance readings were changed to reflect free amino group content using a standard curve obtained with ethylenediamine.

\section{Measurement of the zeta potential}

The zeta potential of CASF was measured in triplicate using a zeta potential analyzer (Malvern Zetasizer Nano ZS90; Malvern Instruments, Malvern, UK) at $25^{\circ} \mathrm{C}$.

\section{Fourier transform infrared measurement}

ASF, CASF (modified with $4 \mathrm{wt} \%$ spermine against $\mathrm{ASF}$ ), ASF + spermine (a mixture of spermine and ASF at a spermine/ASF ratio of 4/100), and spermine solution were dried at $25^{\circ} \mathrm{C}$ and cut into particles $<40 \mu \mathrm{m}$ wide. Approximately $1 \mathrm{mg}$ of these particles was pressed into a pellet with $200 \mathrm{mg}$ of potassium bromide. Fourier transform infrared (FTIR) analysis was performed with a Nicolet 5700 spectrometer. The measurements were taken in the range of $400-4,000 \mathrm{~cm}^{-1}{ }^{36}$

\section{Preparation of CASF/pDNA complexes}

CASF at a concentration of $10 \mathrm{mg} / \mathrm{mL}$ was filtered $(0.22 \mu \mathrm{m}$, EMD Millipore, Billerica, MA, USA). Suspensions of $\mathrm{CASF} / \mathrm{pDNA}$ complexes with weight ratios (CASF to pDNA) of $8 / 1,16 / 1,32 / 1,48 / 1$, and $64 / 1$ were prepared by mixing CASF solutions with pDNA, followed by gentle vortexing for 30 seconds and incubation for 30 minutes at $4{ }^{\circ} \mathrm{C}$. PEI/pDNA $(\mathrm{w} / \mathrm{w}, 3 / 2)^{30}$ was chosen in this study as a control. The preparation method of complex suspension of $\mathrm{PEI} / \mathrm{pDNA}(3 / 2)$ used was similar to that of $\mathrm{CASF} / \mathrm{pDNA}$.

\section{Gel retardation assay}

The binding ability between CASF and pDNA was investigated using agarose gel electrophoresis. Complex suspensions of CASF/pDNA (weight ratios 8/1, 16/1, 32/1, 48/1, and 64/1) and ASF/pDNA (64/1) were prepared according to the procedure described earlier. These complex suspensions were loaded onto a $1.0 \mathrm{wt} \%$ agarose gel with ethidium bromide $(0.5 \mu \mathrm{g} / \mathrm{mL})$ and run with Tris-acetate buffer at $100 \mathrm{~V}$ for 30 minutes. Finally, the results were observed by irradiation under UV light.

\section{Characterization of CASF/pDNA complexes}

The suspensions of CASF/pDNA complexes (weight ratios $32 / 1,48 / 1$, and 64/1) were checked for size and surface charge using a zeta potential analyzer at $25^{\circ} \mathrm{C}$. The morphology of the CASF/pDNA (weight ratio 48/1) complexes was observed with scanning electron microscopy (SEM; Hitachi S-4800; Hitachi Ltd., Tokyo, Japan).

\section{Cell culture}

EA.hy926 cells were cultured in Dulbecco's Modified Eagle's Medium (DMEM; Gibco, Thermo Fisher Scientific, Waltham, MA, USA) supplemented with 10\% fetal bovine serum (FBS, Gibco) and 1\% penicillin/streptomycin (Gibco). The cells were cultured in a $37^{\circ} \mathrm{C}$ incubator with $5 \% \mathrm{CO}_{2}$.

\section{Cytotoxicity assay}

Evaluation of cytotoxicity was performed using the MTT assay. The EA.hy926 cells were seeded in 96-well plates at a density of $5 \times 10^{3}$ cells per well and allowed to attach overnight. Then, the cells were incubated with the complexes in serum-free DMEM. After 4 hours, the medium was replaced with $200 \mu \mathrm{L}$ of fresh DMEM medium containing 10\% FBS for an additional 1 day or 2 days. Thereafter, the medium was removed, and $20 \mu \mathrm{L}$ of MTT solution $(5 \mathrm{mg} / \mathrm{mL}$ in DMEM) was added to each well; the cells were incubated at $37^{\circ} \mathrm{C}$ for 4 hours. Formazan crystals that formed in the living cells were dissolved in $100 \mu \mathrm{L}$ of isopropanol containing $4 \mu \mathrm{L} \mathrm{HCl}$ per well for 30 minutes with slight shaking. Absorbance was determined using a microplate reader (Synergy HT, Bio-Tek, Winooski, VT, USA) at $490 \mathrm{~nm}$. Cell viability was evaluated by calculating the percentage of sample absorbance compared to that of the control after subtracting the blank absorbance. All experiments were repeated three times.

\section{In vitro gene transfection}

The EA.hy926 cells were seeded in $20 \mathrm{~mm}$ cover glass bottom cell culture dishes (NEST, Lot No 2013008) at a density 
of $10^{5}$ cells per dish and allowed to reach $80 \%$ confluence. Complex suspensions of CASF/pDNA (weight ratios 32/1 and 48/1) and PEI/pDNA (3/2) were freshly prepared before transfection, and the medium in each well was exchanged for fresh serum-free DMEM. Complexes were added to the dishes with serum-free DMEM, and the cells were incubated with the complexes for 4 hours. The medium was then removed and replaced with complete medium. After 48 hours, the cells were observed using confocal laser scanning microscopy (FV1000; Olympus, Tokyo, Japan). Levels of GFP expression were determined by flow cytometry analysis. The cells were seeded in six-well plates and transfected using the method described earlier. After 48-hour posttransfection, cells were trypsinized, washed, and resuspended in phosphate-buffered saline. Cell-associated fluorescence was quantitatively determined by green fluorescence emissions measured using a FACSCalibur flow cytometer (BD Biosciences, San Jose, CA, USA). Untransfected EA.hy926 cells were used as background controls.

\section{Statistical analysis}

Data were expressed as the mean \pm standard deviation (SD). Significant differences between groups were tested by oneway analysis of variance. Statistical significance was set in advance to a probability level of 0.05 .

\section{Results}

\section{Characterization of CASF}

As shown in Figure 1, the content of free amino groups in unmodified ASF was $\sim 3.7 \mu \mathrm{mol} / \mathrm{mL}$. After modification with $2 \mathrm{wt} \%$ and $4 \mathrm{wt} \%$ spermine against ASF weight in solution, the concentration of free amino groups in ASF increased

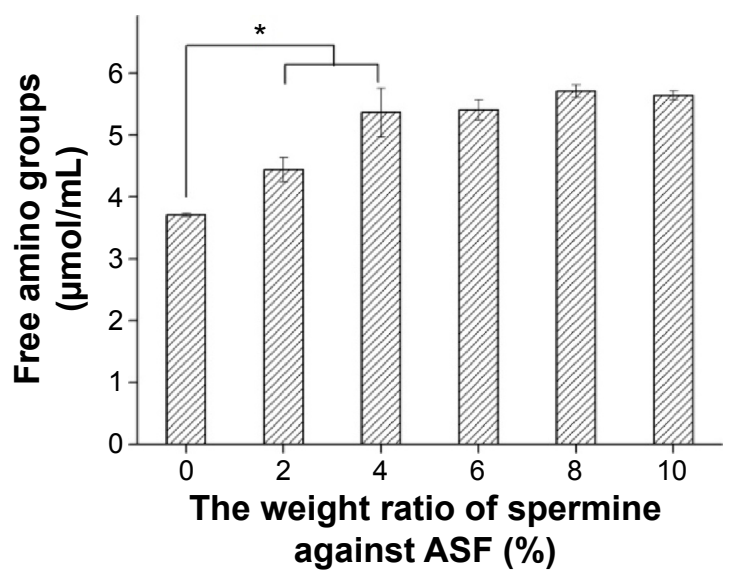

Figure I TNBS assay for the determination of free amino groups in sperminemodified ASF.

Notes: $* P<0.05$ compared with unmodified ASF. The error bars represent the standard deviation of the free amino groups data.

Abbreviations: ASF, Antheraea pernyi silk fibroin; TNBS, trinitrobenzene sulfonic acid. to $\sim 4.4 \mu \mathrm{mol} / \mathrm{mL}$ and $\sim 5.4 \mu \mathrm{mol} / \mathrm{mL}$, respectively. Compared to ASF modified with $4 \mathrm{wt} \%$ spermine, the concentrations of free amino groups in $6 \mathrm{wt} \%, 8 \mathrm{wt} \%$, and $10 \mathrm{wt} \%$ sperminemodified ASF were not significantly different. These results illustrated that using $4 \mathrm{wt} \%$ spermine against ASF to modify ASF can efficiently increase the concentration of free amino groups in ASF side chains.

The zeta potential values of different ratios of spermine against ASF were measured at various weight ratios ranging from $0 \%$ to $10 \%$ (Figure 2). The zeta potential of ASF before modification was $-9.2 \mathrm{mV}$. Using $0.5 \mathrm{wt} \%$ of spermine against ASF changed the negative surface charge of ASF to a positive charge and converted ASF into CASF, at which point the zeta potential of CASF sharply increased to $+1.7 \mathrm{mV}$. When the weight of spermine used against ASF was $2 \%$ or $4 \%$, the zeta potential of CASF continued to increase significantly (to $+5.5 \mathrm{mV}$ and $+6.8 \mathrm{mV}$, respectively). As the weight of spermine against ASF increased, there was no significant effect on the increase in the zeta potential of CASF. The results indicated that using $4 \mathrm{wt} \%$ of spermine against ASF to modify ASF not only increases the concentration of free amino groups in the side chains of ASF but also changes the surface charge of ASF from negative to positive and increases the zeta potential to approximately $+6.8 \mathrm{mV}$. Subsequent experiments used CASF modified with $4 \mathrm{wt} \%$ of spermine against ASF.

FTIR spectra provided further evidence for the successful modification of ASF by spermine. Figure 3 shows the FTIR spectra of CASF, ASF, ASF + spermine, and spermine. The characteristic absorption bands of ASF are at $1,655 \mathrm{~cm}^{-1}$ (amide I), 1,540 $\mathrm{cm}^{-1}$ (amide II), 1,236 $\mathrm{cm}^{-1}$ (amide III), $965 \mathrm{~cm}^{-1}$ (amide IV), and $655 \mathrm{~cm}^{-1}$ (amide V).

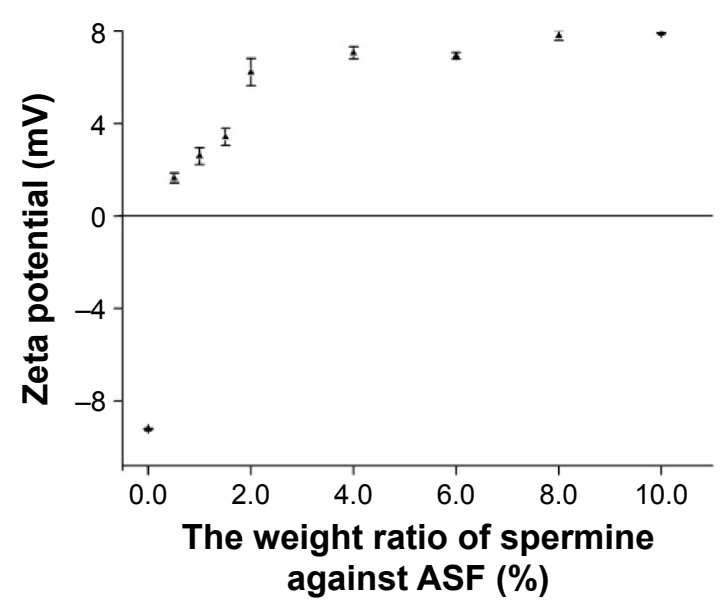

Figure 2 Zeta potential of spermine-modified ASF at different weight ratios of spermine against ASF.

Note: The error bars represent the standard deviation of the zeta potential data. Abbreviation: ASF, Antheraea pernyi silk fibroin. 


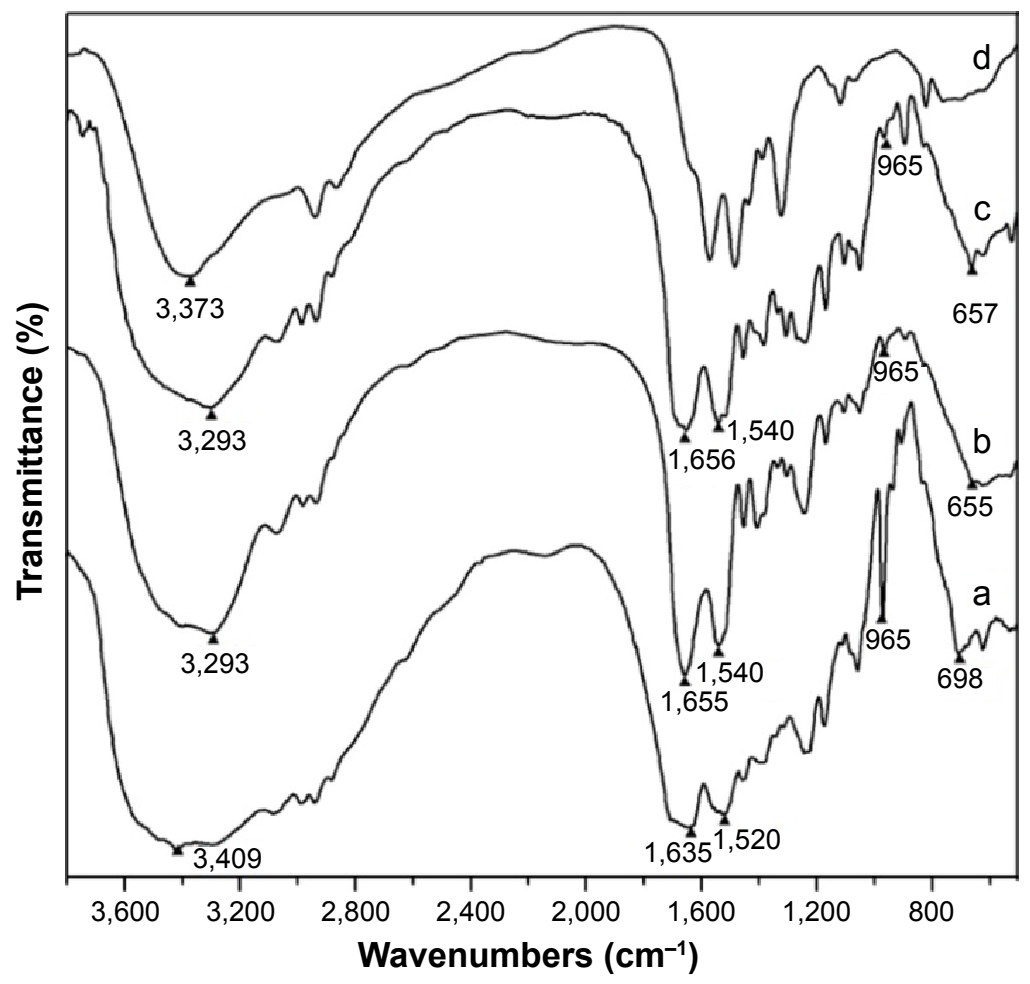

Figure 3 FTIR spectra of the complexes.

Notes: Line graphs represent the following: (a) CASF, (b) ASF, (c) ASF + spermine, and (d) spermine.

Abbreviations: ASF, Antheraea pernyi silk fibroin; CASF, cationized Antheraea pernyi silk fibroin; FTIR, Fourier transform infrared.

Similar absorption bands have been reported previously. ${ }^{37}$ Compared with the spectrum of ASF (Figure 3b), there were no significant changes in the spectrum of the mixture of ASF and spermine (Figure 3c), indicating that spermine mixing with ASF cannot change the structure of side chains of ASF. Several changes were found in the spectrum of CASF (Figure 3a) in contrast with uncationized ASF (Figure 3b). The significant absorption at $965 \mathrm{~cm}^{-1}$ in Figure 3a compared with Figure $3 \mathrm{~b}$ could be attributed to the $\mathrm{O}=\mathrm{C}-\mathrm{N}$ in-plane bending vibration, which was caused by the reaction between $-\mathrm{COOH}$ in the side chains of ASF and $-\mathrm{NH}_{2}$ in spermine. The peak at $3,293 \mathrm{~cm}^{-1}$ in Figure $3 \mathrm{~b}$ shifted to $3,409 \mathrm{~cm}^{-1}$ in Figure 3 a, attributable to the new N-H or $=\mathrm{N}-\mathrm{H}$ symmetrical and asymmetrical stretching vibrations, which appeared near $3,373 \mathrm{~cm}^{-1}$ in the spectrum of spermine (Figure 3d). ${ }^{38-40}$ Compared with unmodified ASF, the amide I and amide II absorption bands of modified ASF at $1,655 \mathrm{~cm}^{-1}$ and $1,540 \mathrm{~cm}^{-1}$ shifted to lower wavenumbers $\left(1,635 \mathrm{~cm}^{-1}\right.$ and $1,520 \mathrm{~cm}^{-1}$, respectively). The amide $\mathrm{V}$ absorption band at $655 \mathrm{~cm}^{-1}$ shifted to a higher wavenumber, $698 \mathrm{~cm}^{-1}$, suggesting that the chemical environment of the amide bonds that were formed by the reaction of $-\mathrm{COOH}$ in the side chains of ASF with the $-\mathrm{NH}_{2}$ in spermine was significantly different from that of the peptide bonds in the main ASF chains. These results demonstrated that ASF was successfully modified by spermine.

\section{Agarose gel retardation assay and morphology of CASF/pDNA complexes}

Negatively charged pDNA was packaged by positively charged CASF via electrostatic interaction to form CASF/ pDNA complexes (Figure 4A). The morphology of the CASF/pDNA (w/w, 48/1) complexes was observed by SEM (Figure 4B). The micrographs show that most complexes were uniform nanospheres $\sim 130-260 \mathrm{~nm}$ in diameter. To investigate the binding capability of CASF to pDNA, an agarose gel retardation electrophoresis assay was performed at different weight ratios of CASF/pDNA (Figure 4C). The DNA band in Lane 2 was similar to that of naked pDNA (Lane 1), which indicated that unmodified ASF cannot package pDNA. However, with an increasing CASF/pDNA weight ratio, a corresponding reduction in migration was observed. When the weight ratio of CASF/pDNA increased to $16 / 1$ (Lane 4), most of the pDNA was retained within the well, with only faint signs of migration; when the weight ratio increased to 32/1, 48/1, or 64/1 (Lanes 5, 6, and 7), there was absolutely no sign of DNA migration. These results suggest that, when the CASF/pDNA weight ratio was equal to or 
A
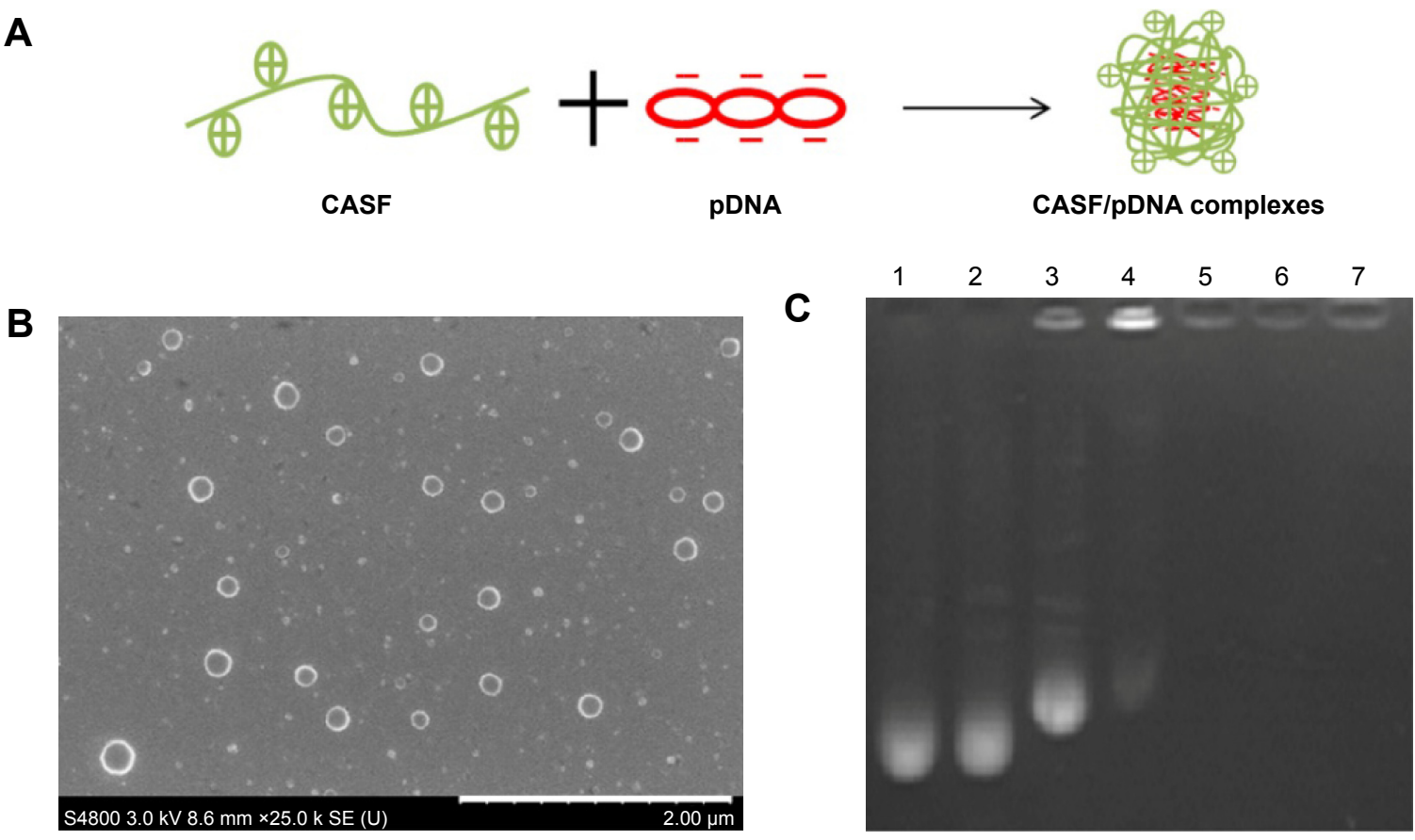

Figure 4 (A) Schematic illustration of the formation of CASF/pDNA complexes. (B) Scanning electron microscopy image of CASF/pDNA complexes with a weight ratio of 48/I. Scale bar: $2 \mu \mathrm{m}$. (C) Agarose gel electrophoresis of CASF/pDNA complexes. Lane I: naked pDNA (control), Lane 2: ASF/pDNA (weight ratio 64/I, control).

Note: Lanes 3, 4, 5, 6, and 7: CASF/pDNA at weight ratios of 8/I, I6/I, 32/I, 48/I, and 64/I, respectively.

Abbreviations: ASF, Antheraea pernyi silk fibroin; CASF, cationized Antheraea pernyi silk fibroin; pDNA, plasmid DNA.

greater than 32/1, CASF could completely package pDNA. Complexes with CASF/pDNA weight ratios of 32/1, 48/1, and 64/1 were chosen for subsequent experiments.

\section{Characterization of CASF/pDNA complexes}

As shown in Table 1, the zeta potentials of three weight ratios of CASF/pDNA complexes were $+3.0 \pm 0.2 \mathrm{mV},+3.2 \pm 0.5 \mathrm{mV}$, and $+3.2 \pm 0.2 \mathrm{mV}$. Compared to $\mathrm{CASF} / \mathrm{pDNA}$ (weight ratio $48 / 1$ ), the zeta potentials of CASF/pDNA (weight ratio 64/1) complexes were not increased. The average diameters of CASF/pDNA complexes at weight ratios of 32/1, $48 / 1$, and $64 / 1$ were $215.4 \pm 4.9 \mathrm{~nm}, 234.7 \pm 5.0 \mathrm{~nm}$, and $280.9 \pm 7.7 \mathrm{~nm}$, respectively. The particle size of CASF/ pDNA complexes at 64/1 weight ratio was the largest of the three weight ratios because excess CASF was absorbed at

Table I The zeta potential $(\mathrm{mV})$ and particle sizes of CASF/ pDNA complexes at different CASF/pDNA weight ratios

\begin{tabular}{lll}
\hline $\begin{array}{l}\text { CASF/pDNA } \\
\text { weight ratio }\end{array}$ & Zeta potential $(\mathrm{mV})$ & Particle size $(\mathrm{nm})$ \\
\hline $32 / \mathrm{I}$ & $+3.0 \pm 0.2$ & $215.4 \pm 4.9$ \\
$48 / \mathrm{I}$ & $+3.2 \pm 0.5$ & $234.7 \pm 5.0$ \\
$64 / \mathrm{I}$ & $+3.2 \pm 0.2$ & $280.9 \pm 7.7$ \\
\hline
\end{tabular}

Abbreviations: CASF, cationized Antheraea pernyi silk fibroin; pDNA, plasmid DNA. the surface of the complexes. Compared with CASF/pDNA complexes of weight ratios $32 / 1$ and $48 / 1$, the zeta potential of CASF/pDNA complexes at weight ratio 64/1 was not increased, but the particle size significantly increased. Small particle sizes lead to higher levels of cellular uptake, ${ }^{41,42}$ and therefore these might be favorable for transfection. In subsequent experiments, the highest weight ratio of modified ASF against pDNA was used (weight ratio 48/1).

\section{Cytotoxicity of CASF/pDNA complexes}

The effects of CASF on the viability of EA.hy926 cells were evaluated by an MTT assay in comparison with PEI (Figure 5). After 1 day of culture, cell viability significantly improved after transfection with CASF/pDNA complexes compared with transfection with PEI/pDNA $(P<0.05)$. The results on the second day were similar to those on the first day, and the cell viability of the CASF/pDNA-transfected EA.hy926 cells was still significantly higher than that of the PEI/pDNA-transfected cells $(P<0.05)$. This result implies the great potential of CASF as a low-toxicity gene delivery carrier.

\section{In vitro transfection of CASF/pDNA complexes}

In this study, $2 \mu \mathrm{g}$ of pDNA was delivered to achieve visible expression after 48 hours. Green fluorescent images 


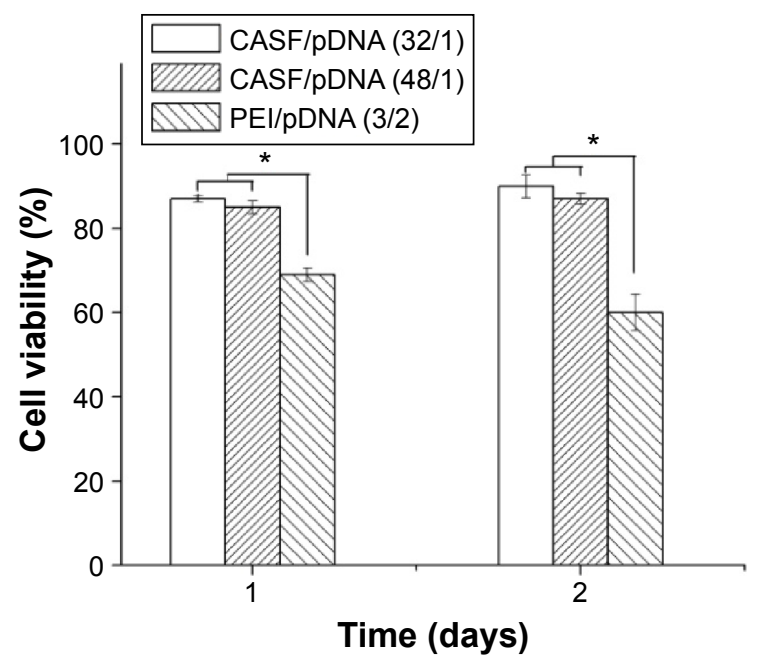

Figure $\mathbf{5}$ The cell viability after treatment of EA.hy 926 cells with CASF/pDNA (at different weight ratios) and PEI/pDNA complexes for I day and 2 days.

Note: $* p<0.05$ compared with PEI/pDNA $(n=3)$.

Abbreviations: CASF, cationized Antheraea pernyi silk fibroin; pDNA, plasmid DNA; PEl, polyethyleneimine.

of transfected EA.hy926 cells were obtained by inversion fluorescence microscopy. As shown in Figure 6, there were greater number of fluorescent cells after transfection with CASF/pDNA complexes (Figure 6A and C) than with PEI/pDNA complexes (Figure 6E). The morphology of the transfected cells was normal, with polygonal characteristics, after transfection with CASF/pDNA complexes (Figure 6B and D), but cells in the PEI/pDNA complex-transfected group appeared to have shrunk and decreased in number (Figure 6F). This result further indicated that CASF possesses lower cytotoxicity than PEI. The transfection efficiency was confirmed by flow cytometry (Figure 7). The transfection efficiencies of the complexes of CASF/pDNA at weight ratio $32 / 1$ and $\mathrm{CASF} / \mathrm{pDNA}$ at weight ratio $48 / 1$ were $15.8 \% \pm 2.0 \%$ and $13.8 \% \pm 2.3 \%$, respectively, higher than the values of $12.0 \% \pm 1.4 \%$ for the PEI/pDNA complexes. The untreated cells showed the lowest levels of transfection efficiency, namely, only $1.6 \% \pm 0.2 \%$.

\section{Discussion}

This is the first report of the synthesis of CASF and its use as a gene delivery carrier. In order to synthesis CASF, ASF whose molecular weight was distributed from $\sim 30 \mathrm{kDa}$ to $\sim 300 \mathrm{kDa}^{43}$ was modified with spermine in the presence of EDC. Carbodiimide coupling is a common method used for the reaction of primary amines with carboxylic acids, resulting in the formation of an amide bond. ${ }^{44}$ ASF contains $\sim 8.66 \mathrm{~mol} \%$ of acidic amino acid residues.$^{45}$ Spermine
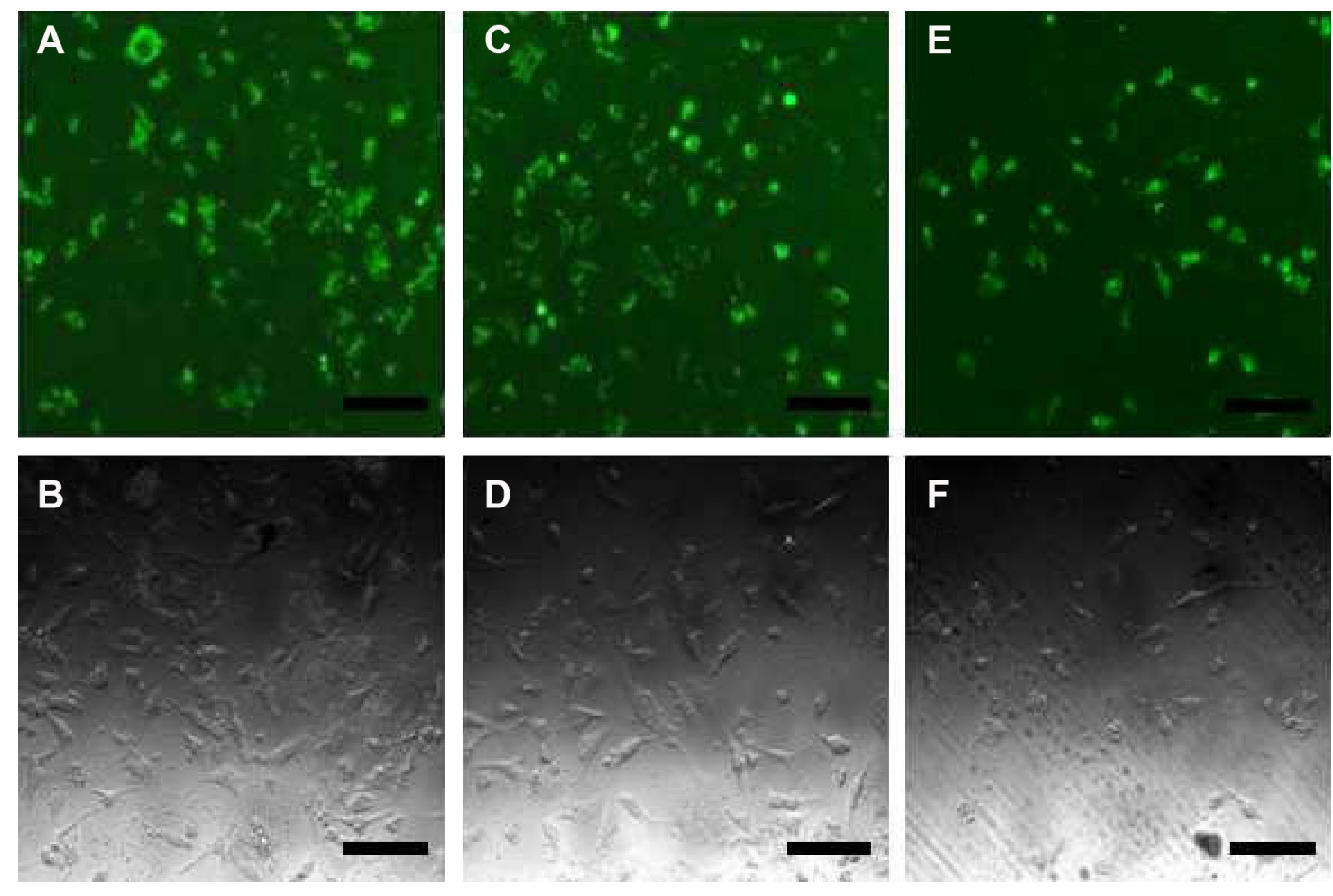

Figure 6 Fluorescence micrographs and cell micrographs of EA.hy 926 cells.

Notes: EA.hy926 cells were transfected with (A, B) CASF/pDNA (at weight ratio of 32/I), (C, D) CASF/pDNA (at weight ratio of 48/I), and (E, F) PEI/pDNA (at weight ratio of $3 / 2$ ). Scale bar is $100 \mu \mathrm{m}$.

Abbreviations: CASF, cationized Antheraea pernyi silk fibroin; pDNA, plasmid DNA; PEl, polyethyleneimine. 
A
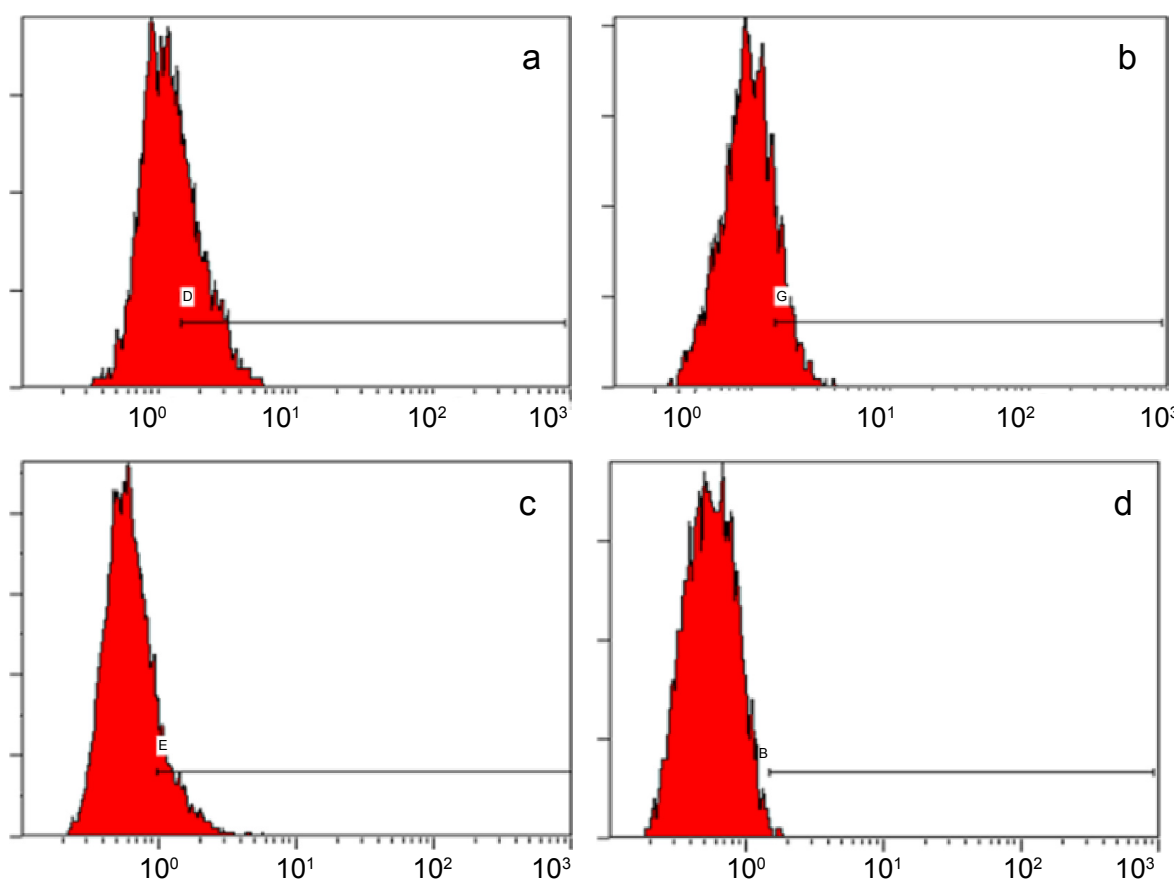

B

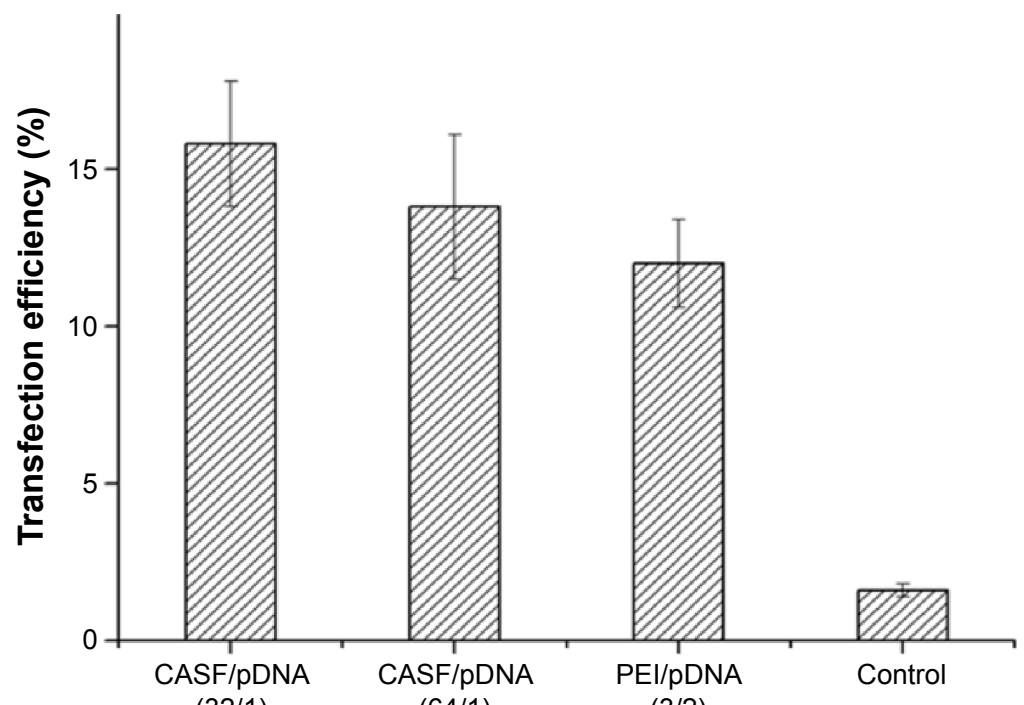

Figure 7 (A) Flow cytometric analysis in EA.hy926 cells after being transfected with CASF/pDNA complexes at weight ratios of (a) 32/I and (b) 48/I, as well as (c) PEl/pDNA complexes at weight ratio of 3/2. (d) Control group (untreated cells). (B) Transfection efficiency of the two complexes at various weight ratios. Note: The values given are mean \pm SD $(n=3)$.

Abbreviations: CASF, cationized Antheraea pernyi silk fibroin; pDNA, plasmid DNA; PEl, polyethyleneimine; SD, standard deviation.

is a polyamine that has two primary and two secondary amino groups. As illustrated in Figure 8, EDC was coupled with the carboxyl groups in the aspartic and glutamic side chains of ASF and formed unstable intermediate urea derivatives ( $O$-acylisourea). Then, $O$-acylisourea was able to react with the primary amine groups in spermine, resulting in the formation of an amide linkage between ASF and spermine to achieve CASF. In this modification process, the self-crosslinking of $-\mathrm{COOH}$ and $-\mathrm{NH}_{2}$ of ASF is a possible reaction. However, the spermine was excessive in the reaction system, and the $-\mathrm{NH}_{2}$ of spermine was dominant around the
$-\mathrm{COOH}$ in ASF side chains; therefore, the $-\mathrm{COOH}$ of ASF would preferentially react with the $-\mathrm{NH}_{2}$ of spermine to form CASF. CASF was purified by dialysis and characterized based on the TNBS assay, zeta potential, and FTIR. These measurements confirmed the introduction of spermine into the side chains of ASF. The TNBS assay provided quantitative evidence that free amino groups had been introduced into the ASF (Figure 1). Zeta potential measurements (Figure 2) revealed that the grafting of spermine brings the zeta potential of ASF from negative to positive. When the weight ratio of spermine against ASF was $>4 \%$, the zeta potential and free 


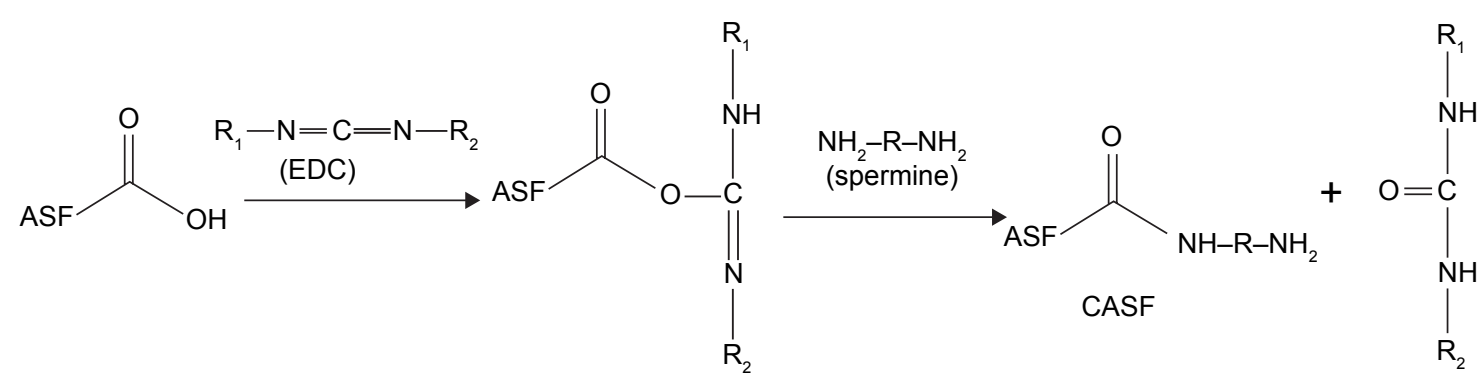

Figure 8 Schematic presentation of modification reaction of ASF with spermine in the presence of EDC.

Abbreviations: ASF, Antheraea pernyi silk fibroin; CASF, cationized A. pernyi silk fibroin; EDC, I-ethyl-(3-dimenthylaminopropyl)-3-carbodiimide hydrochloride.

amino groups were not significantly increased in sperminemodified ASF. The free amino groups and zeta potential of CASF increased significantly as the amount of spermine increased in the initial reaction period. However, with further increase of spermine input amount, on the one hand, the primary amines of spermine reacted with the $-\mathrm{COOH}$ groups in ASF, resulting in the decrease of remaining - $\mathrm{COOH}$ groups. On the other hand, one spermine could react with two $-\mathrm{COOH}$ groups in ASF to form ASF-spermine-ASF because spermine has two primary amines, which accelerated the consumption of $-\mathrm{NH}_{2}$ and $-\mathrm{COOH}$ groups. Consequently, the zeta potential of CASF did not continue to increase. The concentration of free amino groups depended on the content of spermine that was grafted onto the side chains of ASF, but zeta potential was attributed to the ratio of the amines to the carboxyl groups. After modification by spermine, although the increase in amines by spermine grafting was $\sim 35 \%$, the content of carboxyl groups on the ASF surface was obviously decreased. The ratio of the amines to the carboxyl groups significantly increased, which led to the significant increase of zeta potential. FTIR spectra (Figure 3) provided further evidence for the successful synthesis of CASF. The significant absorption at $965 \mathrm{~cm}^{-1}$ in the spectrum of CASF was attributed to the $\mathrm{O}=\mathrm{C}-\mathrm{N}$ in-plane bending vibration, which was caused by the reaction between the $-\mathrm{COOH}$ in the side chains of ASF and the $-\mathrm{NH}_{2}$ in spermine. These results indicated that spermine has been grafted onto the side chains of ASF.

Gene delivery systems based on cationized polymers are significantly affected by the efficiency of DNA condensation. ${ }^{46}$ In this study, positively charged CASF could form complexes with negatively charged pDNA via electrostatic interaction (Figure 4A). In the preparation process of CASF/pDNA nanoparticles, a mixture of the ASF/ spermine/ASF-spermine conjugate/pDNA was probably formed; however, the resulting nanoparticles were mainly $\mathrm{CASF} / \mathrm{pDNA}$ in our experimental system. If the nanoparticles were $\mathrm{ASF} /$ spermine/ASF-spermine conjugate/pDNA, their zeta potential would have been negative. In a previous report, ${ }^{47}$ spermine was shown to bind poorly to DNA, resulting in the loss of DNA protection against nuclease degradation, and could not mediate gene transfer at all. ${ }^{48}$ Thus, the spermine/pDNA complex was not taken as a control in the gel electrophoresis and transfection studies. A gel retardation assay (Figure 4C) showed that CASF was able to package pDNA completely at a weight ratio of 32/1. The CASF displayed a positive charge. Thus, when pDNA combined with the different weight ratios of CASF, the zeta potential would change from a negative charge to a positive charge. The surface zeta potentials of CASF/pDNA complexes in this study were approximately $+3.0 \mathrm{mV}$ to $+3.2 \mathrm{mV}$, which is conducive for the complexes to bind to anionic cell surfaces to facilitate cellular uptake. ${ }^{49}$ Previous studies have indicated that cells typically take up particles with sizes in the range of $\sim 50 \mathrm{~nm}$ to several hundred nanometers. ${ }^{50}$ In this study, the average size of the formed CASF/pDNA complexes is $215-281 \mathrm{~nm}$, which is suitable for transfection. The morphology of the CASF/pDNA weight ratio 48/1 complexes observed by SEM was spherical, with diameters in the range of 130-260 nm. But the average particle size of the CASF/pDNA weight ratio $48 / 1$ complexes determined by Zetasizer was $\sim 234.7 \pm 5.0 \mathrm{~nm}$. Differences in size measurement between SEM imaging and Zetasizer measurements were attributed to the dried state of complexes for SEM measurement versus hydrated state of particles for Zetasizer measurements. Cytotoxicity is a major concern in the application of gene carriers. ${ }^{51} \mathrm{ASF}$ has shown negligible level of toxicity in many cell lines. ${ }^{52-54}$ Moreover, no significant cytotoxicity of pullulan-spermine/ DNA in sensory neurons was detected even at $20 \mathrm{mg} / \mathrm{mL}$ DNA, which is tenfold higher than the concentration conventionally used for transfection. ${ }^{55}$ Hence, in this study, we only investigated the cytotoxicity of CASF/DNA nanoparticles in Figure 5. Compared with PEI/pDNA $3 / 2$ ratio, ${ }^{56}$ the viability of cells transfected with $\mathrm{CASF} / \mathrm{pDNA}$ weight ratio 32/1 and CASF/pDNA weight ratio 48/1 was significantly improved. High molecular weight PEI is relatively cytotoxic because of the high density of its cationic groups and its 
nondegradability. ${ }^{57-59}$ In this study, CASF/pDNA complexes had a low density of positive charges. The zeta potential of the CASF/pDNA complexes was only approximately $+3.0 \mathrm{mV}$ to $+3.2 \mathrm{mV}$, much lower than the $+18.0 \mathrm{mV}$ zeta potential of the $\mathrm{PEI} / \mathrm{pDNA}$ complexes. ${ }^{30}$ The positive charge of the CASF/pDNA complexes was beneficial to enhance the complexes' contact with the cell membrane, and the low density of positive charges avoided destroying the cell membrane permeability when the CASF/pDNA complexes interacted with cells. The biodegradability of CASF allows $\mathrm{CASF} / \mathrm{pDNA}$ complexes to be metabolized and eliminated, thereby showing low cytotoxicity.

The gene transfer ability of the CASF/pDNA complexes was evaluated in EA.hy926 cells. Both qualitative (Figure 6) and quantitative (Figure 7) data revealed that the transfection ability of CASF/pDNA was higher than that of PEI/pDNA. CASF formed by grafting spermine onto ASF not only possessed the ability to package pDNA but also exhibited low cytotoxicity and, therefore, effectively transfected the exogenous gene into cells.

\section{Conclusion}

In this study, spermine was used to modify ASF. The obtained CASF was used to package pDNA, and the resulting CASF/ pDNA complexes were transferred into EA.hy926 cells. Characterizations using the TNBS assay, zeta potential, and FTIR analysis indicated that the $-\mathrm{NH}_{2}$ in spermine effectively reacted with the $-\mathrm{COOH}$ in the side chains of ASF in the presence of EDC. The concentration of free amino groups in CASF was significantly increased via the formation of amide bonds, converting the negative charge of ASF to a positive charge. CASF effectively packaged and condensed pDNA, and the CASF/pDNA complexes exhibited lower cytotoxicity and higher transfection efficiency than PEI/pDNA because CASF possessed excellent cytocompatibility and biodegradability. CASF may be applicable as a novel delivery carrier for clinical gene therapy.

\section{Acknowledgments}

This work was supported by the National Natural Science Foundation of China (31370968, 51403146), the Natural Science Foundation of Jiangsu Province (BK20131177), the College Natural Science Research Project of Jiangsu Province (12KJA430003), and the Priority Academic Program Development of Jiangsu Higher Education Institutions.

\section{Disclosure}

The authors report no conflicts of interest in this work.

\section{References}

1. Ginn SL, Alexander IE, Edelstein ML, Abedi MR, Wixon J. Gene therapy clinical trials worldwide to 2012-an update. J Genet Med. 2013; 15(2):65-77.

2. LaManna CM, Lusic H, Camplo M, McIntosh TJ, Barthélémy P, Grinstaff MW. Charge-reversal lipids, peptide-based lipids, and nucleoside-based lipids for gene delivery. Account Chem Res. 2012;45(7):1026-1038.

3. Robbins PD, Ghivizzani SC. Viral vectors for gene therapy. Pharmacol Ther. 1998;80(1):35-47.

4. Kim T, Bai CZ, Nam K, Park JS. Comparison between arginine conjugated PAMAM dendrimers with structural diversity for gene delivery systems. J Control Release. 2009;136(2):132-139.

5. Guillot-Nieckowski M, Eisler S, Diederich F. Dendritic vectors for gene transfection. New J Chem. 2007;31(7):1111-1127.

6. Numata K, Hamasaki J, Subramanian B, Kaplan DL. Gene delivery mediated by recombinant silk proteins containing cationic and cell binding motifs. J Control Release. 2010;146(1):136-143.

7. Wang W, Nan W, Sun L, Liu WG. A systemic gene vector constructed by zwitterionic polymer modified low molecular weight PEI. Reac Funct Polym. 2013;73(8):993-1000.

8. Ma D, Lin QM, Zhang LM, Liang YY, Xue W. A star-shaped porphyrinarginine functionalized poly (1-lysine) copolymer for photo-enhanced drug and gene co-delivery. Biomaterials. 2014;35(14):4357-4367.

9. Fernandes JC, Qiu X, Winnik FM, et al. Low molecular weight chitosan conjugated with folate for siRNA delivery in vitro: optimization studies. Int J Nanomed. 2012;7:5833-5845.

10. Yu T, Liu X, Bolcato-Bellemin AL, et al. An amphiphilic dendrimer for effective delivery of small interfering RNA and gene silencing in vitro and in vivo. Angew Chem Int Ed Engl. 2012;51(34):8478-8484.

11. Ma Y, Zhang Z, Wang X, Xia WL, Gu HC. Insights into the mechanism of magnetofection using MNPs-PEI/pDNA/free PEI magnetofectins. Int J Pharm. 2011;419(1-2):247-254.

12. Zhang J, Duan Y, Wei D, et al. Co-electrospun fibrous scaffold-adsorbed DNA for substrate-mediated gene delivery. J Biomed Mater Res A. 2011; 96(1):212-220.

13. Deng R, Yue Y, Jin F, et al. Revisit the complexation of PEI and DNA How to make low cytotoxic and highly efficient PEI gene transfection non-viral vectors with a controllable chain length and structure. J Control Release. 2009;140(1):40-46.

14. Goyal R, Tripathi SK, Tyagi S, et al. Gellan gum blended PEI nanocomposites as gene delivery agents: evidences from in vitro and in vivo studies. Eur J Pharm Biopharm. 2011;79(1):3-14.

15. Akinc A, Thomas M,Klibanov AM,Langer R. Exploring polyethyleniminemediated DNA transfection and the proton sponge hypothesis. J Genet Med. 2005;7(5):657-663.

16. Cho SK, Kwon YJ. Polyamine/DNA polyplexes with acid-degradable polymeric shell as structurally and functionally virus-mimicking nonviral vectors. J Control Release. 2011;150(3):287-297.

17. Pan S, Cao D, Fang R, et al. Cellular uptake and transfection activity of DNA complexes based on poly (ethylene glycol)-poly (l-glutamine) copolymer with PAMAM G2. J Mater Chem B. 2013;1(38):5114-5127.

18. Zhao X, Li Z, Pan H, et al. Enhanced gene delivery by chitosan-disulfideconjugated LMW-PEI for facilitating osteogenic differentiation. Acta Biomater. 2013;9(5):6694-6703.

19. Zhang Q, Zhao Y, Yan S, et al. Preparation of uniaxial multichannel silk fibroin scaffolds for guiding primary neurons. Acta Biomater. 2012;8(7): 2628-2638.

20. Lozano-Pérez AA, Rodriguez-Nogales A, Ortiz-Cullera V, et al. Silk fibroin nanoparticles constitute a vector for controlled release of resveratrol in an experimental model of inflammatory bowel disease in rats. Int J Nanomed. 2014;9:4507.

21. George KA, Shadforth A, Chirila TV, et al. Effect of the sterilization method on the properties of Bombyx mori silk fibroin films. Mater Sci Eng C. 2013;33(2):668-674.

22. Zhao Y, Zhao W, Yu S, Guo Y, Gu X, Yang Y. Biocompatibility evaluation of electrospun silk fibroin nanofibrous mats with primarily cultured rat hippocampal neurons. Bio-Med Mater Eng. 2013;23(6):545-554. 
23. Wang Y, Kim UJ, Blasioli DJ, Kim HJ, Kaplan DL. In vitro cartilage tissue engineering with $3 \mathrm{D}$ porous aqueous-derived silk scaffolds and mesenchymal stem cells. Biomaterials. 2005;26(34):7082-7094.

24. Jeong L, Kim MH, Jung JY, Min BM, Park WH. Effect of silk fibroin nanofibers containing silver sulfadiazine on wound healing. Int $J$ Nanomed. 2014;9:5277.

25. Zhu M, Wang K, Mei J, et al. Fabrication of highly interconnected porous silk fibroin scaffolds for potential use as vascular grafts. Acta

26. Yan LP, Oliveira JM, Oliveira AL, Caridade SG, Mano JF, Reis RL. Macro/microporous silk fibroin scaffolds with potential for articular cartilage and meniscus tissue engineering applications. Acta Biomater. 2012;8(1):289-301.

27. Ruoslahti E, Pierschbacher MD. New perspectives in cell adhesion: RGD and integrins. Science. 1987;238(4826):491-497.

28. Minoura N, Aiba S, Higuchi M, Gotoh Y, Tsukada M, Imai Y. Attachment and growth of fibroblast cells on silk fibroin. Biochem Biophys Res Commun. 1995;208(2):511-516.

29. Minoura N, Aiba S, Gotoh Y, Tsukada M, Imai Y. Attachment and growth of cultured fibroblast cells on silk protein matrices. J Biomed Mater Res. 1995;29(10):1215-1221.

30. Ma C, Lv L, Liu Y, et al. Antheraea pernyi silk fibroin for targeted gene delivery of VEGF165-Ang-1 with PEI. Biomed Mater. 2014;9(3): 035015.

31. Liu Y, You R, Liu G, et al. Antheraea pernyi silk fibroin-coated PEI/ DNA complexes for targeted gene delivery in HEK 293 and HCT 116 Cells. Int J Mol Sci. 2014;15(5):7049-7063.

32. Azzam T, Eliyahu H, Makovitzki A, Linial M, Domb AJ. Hydrophobized dextran-spermine conjugate as potential vector for in vitro gene transfection. J Control Release. 2004;96(2):309-323.

33. Jere D, Kim JE, Arote R, et al. Akt1 silencing efficiencies in lung cancer cells by $\mathrm{sh} / \mathrm{si} / \mathrm{ssiRNA}$ transfection using a reductable polyspermine carrier. Biomaterials. 2009;30(8):1635-1647.

34. Tao W, Li M, Zhao C. Structure and properties of regenerated Antheraea pernyi silk fibroin in aqueous solution. Int J Biol Macromol. 2007;40(5): $472-478$

35. Snyder SL, Sobocinski PZ. An improved 2, 4, 6-trinitrobenzenesulfonic acid method for the determination of amines. Anal Biochem. 1975;64(1): 284-288.

36. Deng WW, Cao X, Wang M, et al. Efficient gene delivery to mesenchymal stem cells by an ethylenediamine-modified polysaccharide from mulberry leaves. Small. 2012;8(3):441-451.

37. Li M, Tao W, Kuga S, et al. Controlling molecular conformation of regenerated wild silk fibroin by aqueous ethanol treatment. Polym Adv Technol. 2003;14(10):694-698.

38. Gupta SK, Kumar M, Guldhe A, et al. Design and development of polyamine polymer for harvesting microalgae for biofuels production. Energy Convers Manage. 2014;85:537-544.

39. Kolahdoozan M, Kalbasi RJ, Hossaini M. Synthesis of heterogeneous copper catalyst based on amino-functionalized triazine rings supported by silica-gel for oxidation of alcohols. J Chem. 2013:749723.

40. Deng W, Fu M, Cao Y, et al. Angelica sinensis polysaccharide nanoparticles as novel non-viral carriers for gene delivery to mesenchymal stem cells. Nanomed Nanotechnol. 2013;9(8):1181-1191.

41. Lu F, Wu SH, Hung Y, Mou CY. Size effect on cell uptake in well-suspended, uniform mesoporous silica nanoparticles. Small. 2009;5(12):1408-1413. Biomater. 2014;10(5):2014-2023.

42. Akinc A, Lynn DM, Anderson DG, Langer R. Parallel synthesis and biophysical characterization of a degradable polymer library for gene delivery. J Am Chem Soc. 2003;125(18):5316-5323.

43. You R, Xu Y, Liu Y, Li X, Li M. Comparison of the in vitro and in vivo degradations of silk fibroin scaffolds from mulberry and nonmulberry silkworms. Biomed Mater. 2015;10(1):015003.

44. Murphy AR, Kaplan DL. Biomedical applications of chemicallymodified silk fibroin. J Mater Chem. 2009;19(36):6443-6450.

45. Sezutsu H, Yukuhiro K. Dynamic rearrangement within the Antheraea pernyi silk fibroin gene is associated with four types of repetitive units. J Mol Evol. 2000;51(4):329-338.

46. Kim JH, Minai-Tehrani A, Kim YK, et al. Suppression of tumor growth in H-ras 12V liver cancer mice by delivery of programmed cell death protein 4 using galactosylated poly (ethylene glycol)-chitosan-graftspermine. Biomaterials. 2012;33(6):1894-1902.

47. D'Agostino L, di Pietro M, Di Luccia A. Nuclear aggregates of polyamines are supramolecular structures that play a crucial role in genomic DNA protection and conformation. FEBS J. 2005;272(15):3777-3787.

48. Kloeckner J, Wagner E, Ogris M. Degradable gene carriers based on oligomerized polyamines. Eur J Pharm Sci. 2006;29(5):414-425.

49. Kunath K, von Harpe A, Fischer D, et al. Low-molecular-weight polyethylenimine as a non-viral vector for DNA delivery: comparison of physicochemical properties, transfection efficiency and in vivo distribution with high-molecular-weight polyethylenimine. J Control Release. 2003;89(1):113-125.

50. Liu Y, Reineke TM. Hydroxyl stereochemistry and amine number within poly (glycoamidoamine)s affect intracellular DNA delivery. J Am Chem Soc. 2005;127(9):3004-3015.

51. Yu B, Chen Y, Ouyang C, Huang H, Ji L, Chao H. A luminescent tetranuclear ruthenium (II) complex as a tracking non-viral gene vector. Chem Commu. 2013;49(8):810-812.

52. Zhao C, Wu X, Zhang Q, Yan S, Li M. Enzymatic degradation of Antheraea pernyi silk fibroin 3D scaffolds and fibers. Int $J$ Biol Macromol. 2011;48(2):249-255.

53. Luan XY, Huo GH, Li MZ, Lu SZ, Zhang XG. Antheraea pernyi silk fibroin maintains the immunosupressive properties of human bone marrow mesenchymal stem cells. Cell Biol Int. 2009;33(11):1127-1134.

54. Patra C, Talukdar S, Novoyatleva T, et al. Silk protein fibroin from Antheraea mylitta for cardiac tissue engineering. Biomaterials. 2012; 33(9):2673-2680.

55. Thakor DK, Teng YD, Tabata Y. Neuronal gene delivery by negatively charged pullulan-spermine/DNA anioplexes. Biomaterials. 2009;30(9): 1815-1826.

56. Ma C, Lv L, Yang W, et al. The use of PEI in the targeted gene delivery of VEGF165 and Ang-1. Adv Mater Res. 2014;88(1-883):394-399.

57. Zheng M, Liu Y, Samsonova O, Endres T, Merkel O, Kissel T. Amphiphilic and biodegradable hy-PEI-g-PCL-b-PEG copolymers efficiently mediate transgene expression depending on their graft density. Int J Pharm. 2012;427(1):80-87.

58. Jiang D, Salem AK. Optimized dextran-polyethylenimine conjugates are efficient non-viral vectors with reduced cytotoxicity when used in serum containing environments. Int J Pharm. 2012;427(1):71-79.

59. Tros de Ilarduya C, Sun Y, Düzgüneş N. Gene delivery by lipoplexes and polyplexes. Eur J Pharm Sci. 2010;40(3):159-170.

International Journal of Nanomedicine

\section{Publish your work in this journal}

The International Journal of Nanomedicine is an international, peerreviewed journal focusing on the application of nanotechnology in diagnostics, therapeutics, and drug delivery systems throughout the biomedical field. This journal is indexed on PubMed Central, MedLine, CAS, SciSearch $\AA$, Current Contents ${ }^{\circledR} /$ Clinical Medicine,

\section{Dovepress} Elsevier Bibliographic databases. The manuscript management system is completely online and includes a very quick and fair peer-review system, which is all easy to use. Visit http://www.dovepress.com/ testimonials.php to read real quotes from published authors.
Journal Citation Reports/Science Edition, EMBase, Scopus and the 